Int. J. Electrochem. Sci., 14 (2019) $8494-8505$

International Journal of

ELECTROCHEMICAL

SCIENCE

WWW.electrochemsci.org

\title{
Electrochemical Sensor for Detection of Ascorbic Acid Using a Methylene Blue-Doped Polyimide-Modified Glassy Carbon Electrode
}

\author{
Tian Yang ${ }^{1}$, Yue Wang ${ }^{1, *}$, Yasushi Hasebe ${ }^{2}$, Lin Chen ${ }^{3}$, Zhiqiang Zhang ${ }^{1}$, Zhizhi Hu ${ }^{1, *}$ \\ ${ }^{1}$ School of Chemical Engineering, University of Science and Technology LiaoNing, 185 Qianshan \\ Middle Road, High-tech zone, Anshan, LiaoNing, 114051, China. \\ ${ }^{2}$ Department of Life Science and Green Chemistry, Faculty of Engineering, Saitama Institute of \\ Technology, 1690 Fusaiji, Fukaya, Saitama 369-0293, Japan. \\ ${ }^{3}$ Department of Chemistry and Materials Engineering, Yingkou Institute of Technology, Yingkou, \\ LiaoNing, 115014, China. \\ *E-mail: wangyue@ustl.edu.cn; huzhizhi@163.com
}

doi: $10.20964 / 2019.09 .03$

Received: 8 May 2019 / Accepted: 21 June 2019 / Published: 31 July 2019

\begin{abstract}
Methylene blue (MB)-doped polyimide-modified glassy carbon electrodes were first employed for the detection of ascorbic acid (AA). The fabricated electrode exhibited excellent electrochemical catalytic activities. The structure of the modified electrodes was evaluated by an advanced mineral identification and characterization system (AMICS), high-temperature confocal laser scanning microscopy (HLSM) and atomic force microscopy (AFM). The electrochemical behavior toward ascorbic acid was studied by amperometric i-t curve measurements and cyclic voltammetry. Moreover, the influences of the conditions such as the volume ratio of methylene blue and polyimide, buffer solution $\mathrm{pH}$, applied potential were optimized in this paper. Under the optimized conditions, the sensitivity of the prepared electrode is $27.19 \mu \mathrm{A}^{-1} \cdot \mathrm{M}^{-1} \cdot \mathrm{cm}^{-2}$, with a wide linear range of $3 \mu \mathrm{M}$ to $3000 \mu \mathrm{M}$ and a detection limit of $1.05 \mu \mathrm{M}$. The as-prepared sensor also shows good selectivity, fast response and long lifetime for the determination of AA. This study creates a new application for polyimides in the electrochemical field.
\end{abstract}

Keywords: polyimide, ascorbic acid, methylene blue, electrochemical sensor

\section{$\underline{\text { FULL TEXT }}$}

(C) 2019 The Authors. Published by ESG (www.electrochemsci.org). This article is an open access article distributed under the terms and conditions of the Creative Commons Attribution license (http://creativecommons.org/licenses/by/4.0/). 